\title{
Role of Drp1, a Key Mitochondrial Fission Protein, in Neuropathic Pain
}

\author{
Luiz F. Ferrari, Adrienne Chum, Oliver Bogen, David B. Reichling, and Jon D. Levine \\ Departments of Medicine, and Oral and Maxillofacial Surgery, and Division of Neuroscience, University of California at San Francisco, San Francisco, \\ California 94143-0440
}

While oxidative stress has been implicated in small-fiber painful peripheral neuropathies, antioxidants are only partially effective to treat patients. We have tested the hypothesis that Drp1 (dynamin-related protein 1), a GTPase that catalyzes the process of mitochondrial fission, which is a mechanism central for the effect and production of reactive oxygen species (ROS), plays a central role in these neuropathic pain syndromes. Intrathecal administration of oligodeoxynucleotide antisense against Drp1 produced a decrease in its expression in peripheral nerve and markedly attenuated neuropathic mechanical hyperalgesia caused by HIV/AIDS antiretroviral [ddC $\left(2^{\prime}, 3^{\prime}\right.$-dideoxycytidine)] and anticancer (oxaliplatin) chemotherapy in male Sprague Dawley rats. To confirm the role of Drp1 in these models of neuropathic pain, as well as to demonstrate its contribution at the site of sensory transduction, we injected a highly selective Drp1 inhibitor, mdivi-1, at the site of nociceptive testing on the dorsum of the rat's hindpaw. mdivi-1 attenuated both forms of neuropathic pain. To evaluate the role of Drpl in hyperalgesia induced by ROS, we demonstrated that intradermal hydrogen peroxide produced dose-dependent hyperalgesia that was inhibited by mdivi-1. Finally, mechanical hyperalgesia induced by diverse pronociceptive mediators involved in inflammatory and neuropathic pain — tumor necrosis factor $\alpha$, glial-derived neurotrophic factor, and nitric oxide-was also inhibited by mdivi-1. These studies provide support for a substantial role of mitochondrial fission in preclinical models of inflammatory and neuropathic pain.

\section{Introduction}

Mitochondrial dysfunction has been implicated in neuropathic pain states, including those produced as side effects of drugs used to treat cancer (e.g., the platinum-based chemotherapeutics oxaliplatin and cisplatin) (Flatters and Bennett, 2006; Joseph and Levine, 2006, 2009) and HIV/AIDS (e.g., the nucleoside reverse transcriptase inhibitors) (Dalakas, 2001; Joseph et al., 2004; Osio et al., 2006; Vanotti et al., 2007). The mechanical hyperalgesia associated with these forms of painful peripheral neuropathy is antagonized by inhibitors of mitochondrial functions, including all five mitochondrial electron transport chain complexes (Joseph and Levine, 2006), oxidative stress (Green et al., 2004; Osio et al., 2006; Vanotti et al., 2007), generation of reactive oxygen species (ROS) (Joseph and Levine, 2010), and apoptotic signaling (Joseph and Levine, 2009). In patients, while antioxidants have been shown to have efficacy against symptoms of peripheral neuropathy associated with diabetes (Ziegler et al., 2004; Argyriou et al., 2006; Foster, 2007; Sima, 2007), complex regional pain syndrome type I, and chemotherapy-induced painful

Received May 3, 2011; revised June 23, 2011; accepted June 29, 2011.

Author contributions: L.F.F., O.B., and J.D.L. designed research; L.F.F., A.C., and 0.B. performed research; L.F.F., D.B.R., and J.D.L. wrote the paper.

This study was funded by the NIH. We thank Dr. Robert Gear for assistance with statistical analysis. The authors declare no competing financial interests.

Correspondence should be sent to Dr. Jon D. Levine, Departments of Medicine and Oral Surgery, C-522/Box0440, 521 Parnassus Avenue, University of California, San Francisco, CA 94143-0440. E-mail: Jon.Levine@ucsf.edu. DOI:10.1523/JNEUROSCI.2223-11.2011

Copyright $\odot 2011$ the authors $\quad 0270-6474 / 11 / 3111404-07 \$ 15.00 / 0$ neuropathy (Bureković et al., 2008; Besse et al., 2009; Mijnhout et al., 2010; Pace et al., 2010), these effects have been relatively modest. It has been suggested that this limited efficacy is due to a failure to achieve sufficient antioxidant levels within the mitochondrion (Murphy and Smith, 2007), the major site of ROS generation (Inoue et al., 2003; Brand, 2010). Of note in this regard, antioxidants targeted to the mitochondrion have been developed (Rocha et al., 2010; Li et al., 2011) and are being studied in preclinical models (Gane et al., 2010; Smith and Murphy, 2011).

Oxidative stress causes disturbances in mitochondrial dynamics (e.g., biosynthesis, transport, and fission and fusion), and disturbances in mitochondrial dynamics lead to the increased production of ROS (Bailey, 2003; Green et al., 2004; Lin and Beal, 2006; Cassidy-Stone et al., 2008; Yu et al., 2008; Chen and Chan, 2009; Li et al., 2010ab; Su et al., 2010; Westermann, 2010; Otera and Mihara, 2011; Tan et al., 2011). Mitochondrial fission, in particular, has been shown to mediate pathophysiological production of ROS (Bailey, 2003). Therefore, in the present study we evaluated the role of mitochondrial fission in neuropathic pain states known to be associated with mitochondrial dysfunction (e.g., antiretroviral and anticancer chemotherapy) (Berger et al., 1993; Dalakas, 2001; Quasthoff and Hartung, 2002; Joseph et al., 2004; Joseph and Levine, 2006, 2009), hyperalgesia induced by mediators of inflammatory, and neuropathic pain, and tested the hypothesis that ROS are upstream mediators of dynamin-related protein 1 (Drp1)-dependent mitochondrial fission in models of chemotherapy-induced neuropathic pain. 


\section{Materials and Methods}

Animals. Experiments were performed on adult male Sprague Dawley rats (200-220 g; Charles River). Animals were housed three per cage, under a $12 \mathrm{~h}$ light/dark cycle, in a temperature- and humidity-controlled environment. Food and water were available ad libitum. All nociceptive testing was done between 10:00 A.M. and 4:00 P.M. All experimental protocols were approved by the University of California, San Francisco Committee on Animal Research and conformed to National Institutes of Health Guidelines for the Care and Use of Laboratory Animals. All efforts were made to minimize the number of animals used and their suffering.

Nociceptive behaviors. The nociceptive withdrawal threshold in rat hindpaw was measured using an Ugo Basile Algesymeter, as previously described (Ferrari and Levine, 2010; Ferrari et al., 2010). Briefly, rats were placed in an acrylic cylindrical restrainer with holes through which the hind legs were free to extend. Four measures of nociceptive threshold were taken at $5 \mathrm{~min}$ intervals, and the mean of the last three measures was defined as the nociceptive threshold. The effect of hyperalgesic agents is expressed as the percentage decrease in paw-withdrawal threshold compared with the control paw-withdrawal threshold obtained before drug application. The effect of inhibitors is expressed as the change in pawwithdrawal threshold measured $30 \mathrm{~min}$ after administration of the inhibitor compared with that obtained immediately before its application.

Drugs. Oxaliplatin, 2', $3^{\prime}$-dideoxycytidine (ddC), the selective Drp1 inhibitor mdivi-1 (Cassidy-Stone et al., 2008; Tanaka and Youle, 2008), hydrogen peroxide $\left(\mathrm{H}_{2} \mathrm{O}_{2}\right)$, nerve growth factor (NGF), and epinephrine were purchased from Sigma. Rat recombinant tumor necrosis factor $\alpha$ (TNF $\alpha$ ) was purchased from R\&D Systems, and the nitric oxide (NO) donor ( \pm )-(E)-ethyl-2-[(E)-hydroxyimino]-5-nitro-3-hexeneamide (NOR-3) and the glial-derived neurotrophic factor (GDNF) were purchased from EMD Chemicals. Intradermal injections of experimental agents were administered via a beveled 30 gauge hypodermic needle attached by PE-10 polyethylene tubing to a microsyringe (10 $\mu$ l; Hamilton). Oxaliplatin and ddC were dissolved in $0.9 \% \mathrm{NaCl}$ immediately before intravenous injection, mdivi-1 was dissolved in DMSO (100\%), and $\mathrm{H}_{2} \mathrm{O}_{2}$ was diluted in distilled water. Stock solutions of NGF and GDNF $(1 \mu \mathrm{g} / \mu \mathrm{l}$ dissolved in $0.9 \% \mathrm{NaCl}$ containing $0.5 \% \mathrm{BSA}$ and stored at $-20^{\circ} \mathrm{C}$ ), TNF $\alpha$ (dissolved in PBS $0.1 \%$ BSA), and NOR-3 (dissolved in $100 \% \mathrm{DMSO}$ ) were diluted in $0.9 \% \mathrm{NaCl}$ immediately before injection. Epinephrine was dissolved in distilled water with an equivalent amount of ascorbic acid immediately before injection. The effect of each chemical was determined in different groups of rats.

ddC-induced neuropathy. Nucleoside reverse transcriptase inhibitors (NRTIs), used extensively in the treatment of HIV/AIDS, induce painful peripheral neuropathy, which we have modeled in the rat (Joseph et al., 2004). A single dose of the NRTI ddC (50 mg/kg, i.v.) produces a significant reduction in nociceptive threshold from day 1 after its administration, which persists for $>20 \mathrm{~d}$ (Joseph et al., 2004).

Oxaliplatin-induced neuropathy. The cancer chemotherapeutic agent oxaliplatin was dissolved in normal saline, and its volume was adjusted to $1 \mathrm{ml} / \mathrm{kg}$ for administration into a tail vein $(2 \mathrm{mg} / \mathrm{kg}$, i.v. $)$ followed by a bolus injection of an equal volume of saline via the same injection needle. Paw-withdrawal threshold was found to be lowered on the 6th and 15th day after oxaliplatin injection.

Oligodeoxynucleotide antisense to Drp1. The oligodeoxynucleotide (ODN) antisense to Drp1 mRNA, 5'CCA CTA CGA CAA TCT GAG GC3' (Invitrogen), was directed against a unique region of the rat mRNA sequence. The corresponding EMBL GenBank accession number and ODN position within the mRNA sequence are AF019043 and 812 to 831, respectively. This sequence should block the translation of all six known isoforms (according to UniProtKB/Swiss-Prot data base entry O35303). The ODN mismatch sequence 5'ACA CTT CTA CAT TCG GAC GC3' corresponds to the Drp1 antisense sequence with six bases mismatched (denoted in bold).

ODNs were reconstituted in nuclease-free $0.9 \% \mathrm{NaCl}$ to a concentration of $10 \mu \mathrm{g} / \mu \mathrm{l}$ and stored at $-20^{\circ} \mathrm{C}$ until use. For each injection, rats were anesthetized with $2.5 \%$ isoflurane. A dose of $40 \mu \mathrm{g}$ (injection volume $20 \mu \mathrm{l}$ ) of Drp1 ODN antisense or mismatch was administered using an insulin syringe with a 29 gauge needle inserted intrathecally, on the midline between the fourth and fifth lumbar vertebrae, once daily for 3 consecutive days.

Protein extraction and Western blot. To confirm that the ODN antisense to Drp1 mRNA produced a decrease in the protein expression level of Drp1 in primary afferent fibers, a Western blot analysis was performed. Saphenous nerves from anesthetized rats were ligated with 4-O silk surgical suture, $1 \mathrm{~cm}$ above the knee-level bifurcation of the sciatic nerve. A $5 \mathrm{~mm}$ section of the saphenous nerve proximal to the ligation was harvested $24 \mathrm{~h}$ after the last injection of ODN, and protein extraction, SDS-PAGE gel electrophoresis, and Western blot analysis were performed as previously described (Bogen et al., 2008; Summer et al., 2008).

Drp1 immunoreactivity was revealed with 1:500 affinity-purified rabbit anti-Drp1 antibody (NB110-55288; Novus Biologicals) followed by 1:1000 horseradish peroxidase-conjugated donkey anti-rabbit antibody (NA-934; GE Healthcare Biosciences). Immunoreactivities of the protein samples were normalized using 1:1000 affinity-purified rabbit anti- $\beta$-actin antibody (ab8227; Abcam) followed by 1:5000 horseradish peroxidaseconjugated donkey anti-rabbit antibody (GE Healthcare Biosciences). Immunoreactivity was visualized using an enhanced chemiluminescence detection system (Pierce Biotechnology). Results were analyzed by computer-assisted densitometry, and levels of Drp1 immunoreactivity were normalized with respect to $\beta$-actin levels in each sample. The percentage decrease in protein expression level was calculated as follows: [normalized density for antisense - normalized density for mismatch]/ normalized density for mismatch $\times 100$.

Statistics. In all behavioral experiments, the dependent variable was paw-withdrawal threshold, expressed as the percentage change from baseline. One-way between-subjects ANOVA or two-way repeatedmeasures ANOVA was performed, as appropriate. In cases where more than two groups were compared, Scheffé's post hoc was used to determine the basis of significant differences. Student's $t$ tests were performed for the data shown in the lower part of Figure $1 A$ and in Figure 5. Data are presented as mean \pm SEM.

\section{Results}

\section{Role of Drp1 in ddC and oxaliplatin neuropathy}

To evaluate the role of Drp1 in neuropathic pain, two very different, but complementary, methods were used to block Drp1 function, as follows: (1) intrathecal administration of an ODN designed to be antisense to Drp1 mRNA, to produce a decrease in its expression level in the primary afferent nociceptor; and (2) intradermal injection of the mitochondrial division inhibitor mdivi-1 at the site of nociceptive testing, to both confirm a role of Drp1-mediated mitochondrial fission as well as to evaluate its contribution in the peripheral terminal of the primary afferent nociceptor.

Spinal intrathecal injection of ODN antisense for mRNA encoding Drp1, $40 \mu \mathrm{g} / \mathrm{d}$ for 3 consecutive days, produced a $24 \%$ decrease in the level of Drp1 in the saphenous nerve (ODN antisense, $N=6$; compared with ODN mismatch, $N=6 ; p=0.027$ ) (Fig. 1A). Of note, since Drp1 is present in all cells in the nerve, this value must underestimate the attenuation of Drp1 expression within the primary afferent nerve fibers. This protocol for Drp1 ODN antisense administration decreased ddC-induced mechanical hyperalgesia (DRP1 AS; $N=6$ ) (Fig. $1 B$ ) by $76.8 \%$, when compared with the effect of ODN mismatch (DRP1 MM; $N=6$ ) (Fig. $1 B$ ).

To confirm the contribution of Drp1 to ddC-induced mechanical hyperalgesia as well as to demonstrate that the site of action of Drp1 in the inhibition of neuropathic pain is the peripheral terminal of the primary afferent nociceptor, we evaluated the effect of intradermal injection of the selective Drp1 inhibitor mdivi-1 on ddC-induced mechanical hyperalgesia. We found that mdivi-1 produced a dose-dependent inhibition of ddCinduced hyperalgesia $(0-10 \mu \mathrm{g} / \mathrm{paw}, N=6, p<0.001)$ (Fig. $2 A)$ and at the highest dose $(10 \mu \mathrm{g})$ markedly reversed the neuro- 

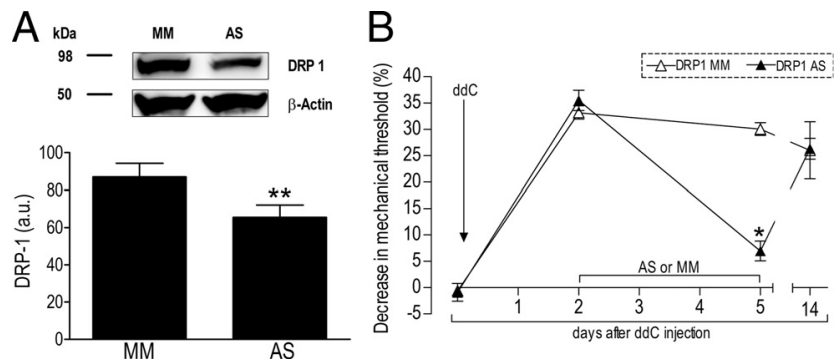

Figure 1. Role of Drp1 in ddC-induced neuropathic pain. $A$, Knockdown of Drp1. Analyzing the expression of Drp1 in rats treated with ODN MM or AS for Drp1 mRNA demonstrated a significant reduction of Drp1 protein levels in the saphenous nerve; $\mathrm{MM}=87 \pm 7 ; \mathrm{AS}=66 \pm$ 7 arbitrary units normalized to the reference protein $\beta$-actin $\left(N=6\right.$; ${ }^{* *} p=0.027$, unpaired Student's $t$ test) showed a $24 \%$ decrease in Drp1 protein. Of note, the calculated molecular weights of Drp1 and $\beta$-actin are 84 and $42 \mathrm{kDa}$, respectively. $\boldsymbol{B}$, Attenuation of chemotherapyinduced hyperalgesia by Drp1 ODN antisense. Rats were treated with a single intravenous injection of ddC ( $50 \mathrm{mg} / \mathrm{kg}$ ). $2 \mathrm{~d}$ later, ODN antisense (DRP1 AS, $\mathbf{\Delta}$ ) or mismatch (DRP1 MM, $\triangle$ ) $(40 \mu \mathrm{g})$ for Drp1 mRNA was injected intrathecally on 3 consecutive days. Mechanical nociceptive threshold was measured, by the Randall-Selitto paw-withdrawal test, on the second and fifth day post-ddC injection. $N=6$ paws for both groups. One-way ANOVA showed a significant difference between the AS and MM groups on the fifth day $\left(F_{(1,10)}=141.960 ;{ }^{*} p<0.001\right)$.
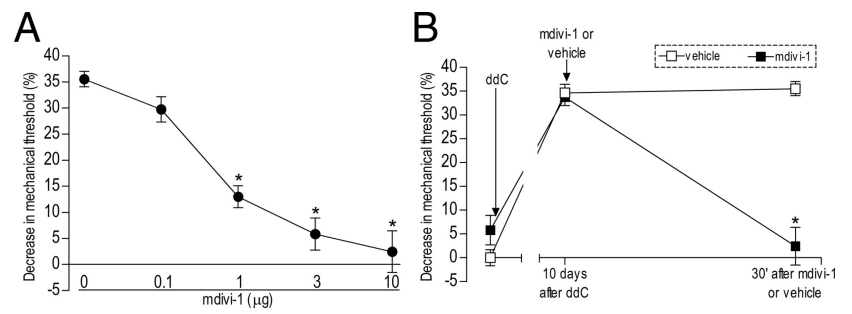

Figure 2. Effect of local injection of Drp1 inhibitor mdivi-1 on ddC-induced hyperalgesia. $A$, Dose-response curve for the effect of intradermal mdivi-1 on the hyperalgesia induced by intravenous injection of $d d C$. Rats received a single injection of $\mathrm{ddC}(50 \mathrm{mg} / \mathrm{kg})$. Ten days later mdivi-1, $(0,0.1,1,3$, and $10 \mu \mathrm{g})$ was administered in separate groups. Mechanical thresholds were tested $30 \mathrm{~min}$ after mdivi-1 administration. One-way ANOVA showed a significant difference $\left(F_{(4,25)}=1287.841 ; p<0.001\right)$. Scheffé's post hoc tests showed that the effects of all doses except $0.1 \mu \mathrm{g}$ were significantly different $\left({ }^{*} p<0.001\right)$ from the control group $(0 \mu \mathrm{g}$, vehicle); however, there was no significant difference in the effects of doses between 3 and 10 $\mu \mathrm{g}(p=0.151$ and 0.941 , respectively); therefore, we chose the $3 \mu \mathrm{g}$ dose for this study. $\boldsymbol{B}$, Attenuation of ddC-induced hyperalgesia by mdivi-1. For this experiment, the maximal dose of mdivi-1 $(10 \mu \mathrm{g} ; n=6)$ or vehicle $(n=6)$ was administered $10 \mathrm{~d}$ after ddC injection in separate groups of rats. Mechanical thresholds were tested before and $30 \mathrm{~min}$ after injections. As shown in $\boldsymbol{A}$, mdivi-1 ( $\square$ ) attenuated ddC-induced hyperalgesia, but vehicle $(\square)$ had no effect. The two-way repeated-measures ANOVA showed a significant main effect of group $\left(F_{(1,10)}=\right.$ $\left.60.503 ;{ }^{*} p<0.001\right)$, a significant group $\times$ time interaction $\left(F_{(1,10)}=35.133 ;{ }^{*} p<0.001\right)$, as well as a significant main effect of time $\left(F_{(1,10)}=31.385 ;{ }^{*} p<0.001\right)$.

pathic mechanical hyperalgesia (Fig. $2 \mathrm{~B}$ ). Neither the ODN antisense for Drp1 nor the mitochondria division inhibitor mdivi-1 induced changes in the baseline mechanical threshold by itself (data not shown).

We also evaluated the role of Drp1 in another model of mitochondrial function-dependent painful peripheral neuropathy, namely, that induced by the commonly used platinum-based cancer chemotherapeutic agent oxaliplatin (Gamelin et al., 2002; Lehky et al., 2004; Tournigand et al., 2004; Kelland, 2007; Joseph and Levine, 2009). Injection of oxaliplatin (2 mg/kg, i.v.) produced a significant decrease in mechanical nociceptive threshold (Joseph et al., 2008). In this form of chemotherapy-induced painful peripheral neuropathy, which is thought to have two distinct phases (Joseph et al., 2008; Joseph and Levine, 2009), intradermal mdivi-1 (3 $\mu \mathrm{g} / \mathrm{paw})$ inhibited mechanical hyperalgesia in both
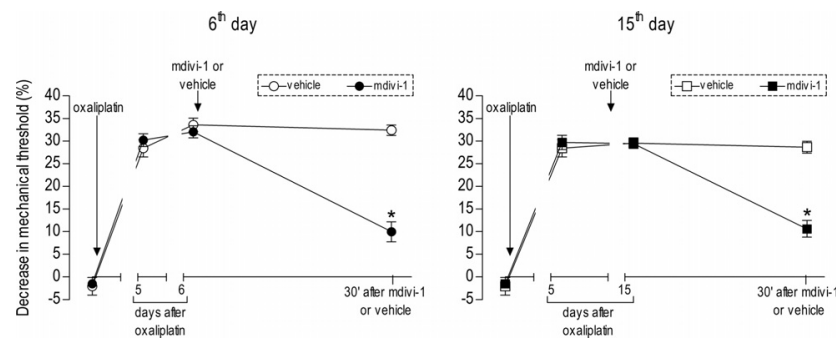

Figure 3. Effect of the Drp1 inhibitor on early and late phases of oxaliplatin-induced neuropathy. A single dose of oxaliplatin ( $2 \mathrm{mg} / \mathrm{kg}$ ) was administered intravenously. Intradermal injection of mdivi-1 ( $3 \mu \mathrm{g}, n=6)$ or vehicle $(n=6)$ was performed on the sixth day (first phase, left) or on the 15th day (second phase, right) post-oxaliplatin injection. Mechanical thresholds were tested before and 30 min after mdivi-1 $(\mathbf{O}$ and $\mathbf{\square})$ or vehicle $(\bigcirc$ and $\square)$ administration. mdivi-1 significantly attenuated both phases of oxaliplatin-induced hyperalgesia. For early-phase hyperalgesia, a two-way repeated-measures ANOVA showed a significant main effect of group $\left(F_{(1,20)}=64.590 ;{ }^{*} p<0.001\right)$, a significant group $\times$ time interaction $\left(F_{(1,20)}=50.641 ;{ }^{*} p<0.001\right)$, and a significant main effect of time $\left(F_{(1,20)}=48.010 ;{ }^{*} p<\right.$ $0.001)$. For late-phase hyperalgesia, there was also a significant main effect of group $\left(F_{(1,8)}=\right.$ $\left.35.666 ;{ }^{*} p<0.001\right)$, a significant group $\times$ time interaction $\left(F_{(1,8)}=41.183 ;{ }^{*} p<0.001\right)$, and a significant main effect of time $\left(F_{(1,8)}=49.882 ;{ }^{*} p<0.001\right)$.

phases (both $p<0.001$ ), with phase 1 evaluated on the 6th day and phase 2 on the 15th day after injection of oxaliplatin (Fig. 3).

\section{Role of Drp1 in the hyperalgesia induced by reactive oxygen species}

Since several cancer chemotherapeutic agents (including oxaliplatin) and HIV/AIDS therapies (including the NRTI ddC) are thought to produce their side effects by generation of ROS (Skuta et al., 1999; Kim et al., 2008), and, since we have shown that antioxidants inhibit painful peripheral neuropathy induced by ddC (Joseph and Levine, 2006) and oxaliplatin (Joseph and Levine, 2009) by action at the peripheral terminal of the primary afferent nociceptor, we determined next whether intradermal injection of the Drp1 inhibitor mdivi-1 also reverses mechanical hyperalgesia induced by ROS in the peripheral terminal of the primary afferent nociceptor.

We first developed a model to determine whether ROS can act at the peripheral terminal of the primary afferent nociceptor to produce mechanical hyperalgesia. Local administration of hydrogen peroxide, a well established method for producing an increase in intracellular ROS (Skjelbakken et al., 1996), produces a dose-dependent decrease in mechanical nociceptive threshold $(N=6, p<0.001)$ (Fig. $4 A)$. The Drp1 inhibitor mdivi-1 $(3 \mu \mathrm{g})$ markedly attenuated the effect of a concentration of hydrogen peroxide ( $5 \mu \mathrm{l}$ of a $1 \%$ solution) that produced maximal hyperalgesia $(p<0.009)$ (Fig. $4 B$ ). Thus, the enhanced production of ROS, which is thought to mediate the toxic side effects of HIV/ AIDS and cancer chemotherapy drugs, itself produces mitochondrial fission-dependent mechanical hyperalgesia.

\section{Role of Drp1 in pronociceptive mediator-induced hyperalgesia}

Finally, we performed experiments to determine whether Drp1 plays a role in mechanical hyperalgesia induced by pronociceptive mediators implicated in inflammatory and neuropathic pain, including cytokines and growth factors, as well as to confirm the contribution of mitochondria at the site of sensory transduction in the peripheral terminal of the primary afferent nociceptor. We evaluated the ability of mdivi-1, injected intradermally at the site at which the mediator was injected and nociceptive testing was performed, to inhibit mechanical hyperalgesia induced by intra- 
A

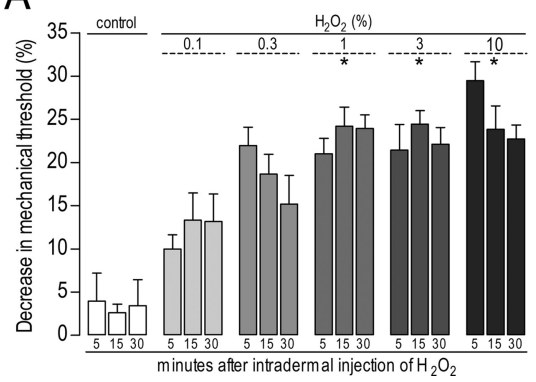

B

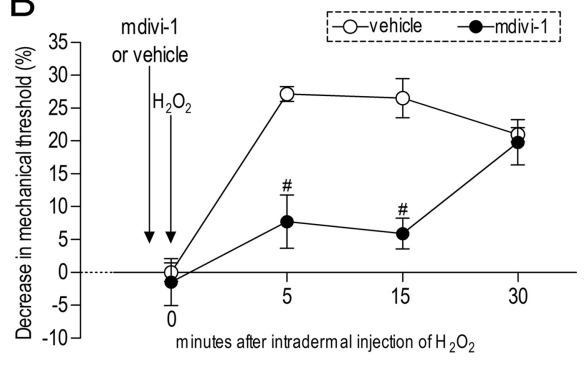

Figure 4. Hydrogen peroxide $\left(\mathrm{H}_{2} \mathrm{O}_{2}\right)$-induced mechanical hyperalgesia is dependent on Drp1 activation. $A$, Increasing doses of $\mathrm{H}_{2} \mathrm{O}_{2}$ (control, $0.1,0.3,1,3,10 \%$ ) were administered intradermally into the dorsum of the hindpaws in separate groups of rats. Testing was performed 5,15 , and 30 min later. Higher doses of $\mathrm{H}_{2} \mathrm{O}_{2}$ induced significant hyperalgesia at all three time points. The two-way repeated-measures ANOVA showed a significant main effect of group $\left(F_{(5,28)}=21.351 ; p<0.001\right)$, but neither the main effect of time $\left(F_{(2,56)}=775 ; p=0.466\right)$ nor the group $\times$ time interaction $\left(F_{(2,56)}=1.885 ; p<0.067\right)$ were significant. Scheffé's post hoc tests showed a significant difference between the control and the three highest $\mathrm{H}_{2} \mathrm{O}_{2}$ groups ( ${ }^{*} p<0.001$ for all three) but not the group that received $0.1 \% \mathrm{H}_{2} \mathrm{O}_{2}(p=0.115)$. $\boldsymbol{B}$, Effect of mdivi-1 ( $\left.3 \mu \mathrm{g} / \mathrm{paw}\right)$ on $\mathrm{H}_{2} \mathrm{O}_{2}$-induced hyperalgesia. mdivi- $1(\boldsymbol{O}$, $3 \mu \mathrm{g}, n=6$ ) or vehicle $(\mathrm{O}, n=6)$ was administered 5 min before $\mathrm{H}_{2} \mathrm{O}_{2}$ in separate groups of rats. Testing was performed 5,15 , and 30 min after $\mathrm{H}_{2} \mathrm{O}_{2}(1 \%)$ administration into the same site. mdivi-1 attenuated $\mathrm{H}_{2} \mathrm{O}_{2}$-induced hyperalgesia. A two-way repeated-measures ANOVA showed a significant main effect of group $\left(F_{(6.5)}=11.126 ;{ }^{\#} p=0.009\right)$, a significant group $\times$ time interaction $\left(F_{(12,10)}=5.578 ; p=0.013\right)$, and a significant main effect of time $\left(F_{(2,10)}=17.522 ; p=0.002\right)$.
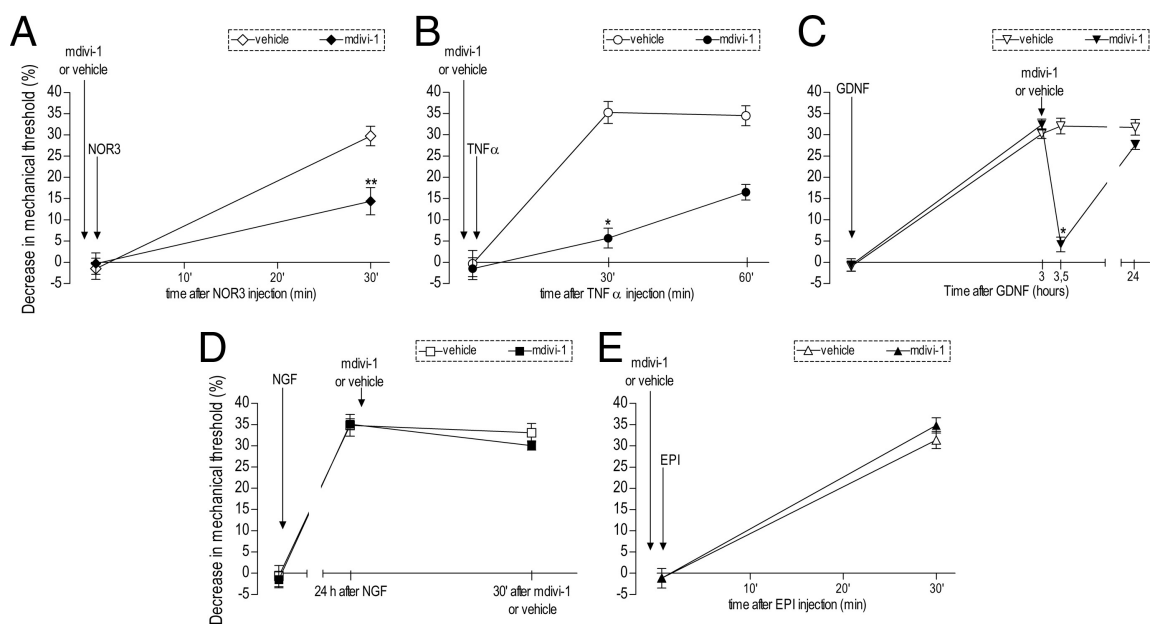

Figure 5. Involvement of Drp1 in the mechanical hyperalgesia induced by mediators implicated in inflammatory and neuropathic pain. The effect of mdivi-1 (3 $\mu \mathrm{g})$ was evaluated in five different models of acute hyperalgesia, including NO (A), $\operatorname{TNF} \alpha(\boldsymbol{B})$, $\operatorname{GDNF}(\boldsymbol{C}), \operatorname{NGF}(\boldsymbol{D})$, and epinephrine (EPI) (E). mdivi-1 (full symbols) or vehicle (empty symbols) was injected 10 min before the nitric oxide donor NOR-3 $(1 \mu \mathrm{g})$, TNF $\alpha(0.1 \mu \mathrm{g})$, or EPI $(0.1 \mu \mathrm{g})$. Rats treated with GDNF $(10 \mathrm{ng})$ or NGF $(1 \mu \mathrm{g})$ received mdivi-1 or vehicle 1 or $24 \mathrm{~h}$ later, respectively. Mechanical thresholds were evaluated $30 \mathrm{~min}$ after mdivi-1/vehicle injection by the Randall-Selitto paw-withdrawal test. Student's t tests showed significant attenuation of the mechanical hyperalgesia induced by $\operatorname{NOR-3}\left(\diamond, t_{(10)}=3.902 ;{ }^{* *} p=0.003\right), \operatorname{TNF} \alpha\left(\bullet, t_{(10)}=8.474 ;{ }^{*} p<0.001\right), \operatorname{and} \operatorname{GDNF}\left(\boldsymbol{\nabla}, t_{(10)}=10.953 ;{ }^{*} p<0.001\right)$ in the groups treated with mdivi-1. No significant effect of mdivi-1 was observed on NGF- or EPI-induced hyperalgesia $\left(\square, t_{(10)}=1.307\right.$; $p=0.220$ and $\mathbf{\Lambda}, t_{(10)}=1.281 ; p=0.229$, respectively). $N=6$ for all groups.

dermal injection of NGF (Malik-Hall et al., 2005), TNF $\alpha$ (Parada et al., 2003), the NO donor NOR-3 (Aley et al., 1998), and epinephrine (Leene et al., 1976). While intradermal injection of mdivi-1 $(3 \mu \mathrm{g})$ significantly inhibited hyperalgesia induced by NOR-3 $(1 \mu \mathrm{g} ; N=6, p=0.003)$, TNF $\alpha(0.1 \mu \mathrm{g} ; N=6, p<0.001)$, and $\operatorname{GDNF}(10 \mathrm{ng} ; N=6, p<0.001)$, it did not significantly inhibit hyperalgesia of similar magnitude induced by NGF $(1 \mu \mathrm{g} ; N=$ $6, p=\mathrm{NS})$ or epinephrine (0.1 $\mu \mathrm{g} ; N=6, p=\mathrm{NS})$ (Fig. 5).

\section{Discussion}

Many of the side effects of chemotherapeutic agents used in the treatment of cancer and HIV/AIDS, including their neurotoxic effects on the peripheral nervous system, are thought to be, at least in part, the consequence of oxidative stress (Joseph et al., 2008; Kallianpur and Hulgan, 2009), which in turn leads to overproduction of ROS (Mondal et al., 2004; Gogvadze et al., 2009) and neurotoxicity. Clinical trials with antioxidants, however, have reported only a relatively modest inhibition of symptoms in patients with diverse forms of painful peripheral neuropathies (Ziegler et al., 2004, 2006; Chen et al., 2009), suggesting the possibility that therapeutic interventions targeted upstream to the production of ROS might be more effective. Since mitochondrial fission may be necessary for the overproduction of ROS (Su et al., 2010), we evaluated the hypothesis that mitochondrial fission plays a role in the ROS-dependent pain symptoms associated with chemotherapy-induced neuropathies.

We found that spinal intrathecal administration of ODN antisense to Drp1 attenuated ddC-induced mechanical hyperalgesia. Similar to the effect of Drp1 antisense, intradermal injection of the selective Drp1 inhibitor mdivi-1 markedly attenuated the mechanical hyperalgesia induced by ddC. Of note, mdivi-1 displays a high degree of selectivity for dynamins involved in mitochondrial fission, having no effect even on other dynamin-related proteins (e.g., mitochondrial fusion dynamins and the endocytic dynamin) (Lackner and Nunnari, 2010).

To demonstrate that Drp1 is involved in mechanical hyperalgesia associated with other forms of painful peripheral neuropathy induced by neurotoxic agents that are also thought to produce their toxic side effects secondary to generation of ROS, we evaluated the effect of mdivi-1 on the mechanical hyperalgesia associated with oxaliplatin-induced peripheral neuropathy (Joseph and Levine, 2009). Again, mdivi-1 markedly attenuated the mechanical hyperalgesia in oxaliplatin-induced peripheral neuropathy. Of note, mdivi-1 has been shown previously to be efficacious in rodent models of renal damage induced by another platinum-based chemotherapeutic agent, cisplatin (Brooks et al., 2009; Lackner and Nunnari, 2010), which also produces painful peripheral neuropathy as a clinically important dose-limiting side effect (Peltier and Russell, 2002).

Since oxidative stress induces mitochondrial fragmentation (i.e., fission) (Wu et al., 2011), we tested for a role of mitochondrial fission in the mechanical hyperalgesia induced by excessive ROS. In these experiments, we evaluated the effect of mdivi-1 on hydrogen peroxide-induced mechanical hyperalgesia. One of the best recognized effects of hydrogen peroxide in the cell is in the generation of oxidative stress (Stone and Yang, 2006). Of note, hydrogen peroxide can be produced in response to, among other stimuli, cytokines and growth factors, and is found in concentrations up to $1 \mathrm{~mm}$ in diseased tissue (and extracellular administra- 
tion of hydrogen peroxide mimics the generation of hydrogen peroxide by cytokines and growth factors) (Veal et al., 2007). Furthermore, it has been shown that mutations in Drp1 that produce a decrease in its function display resistance to hydrogen peroxide-induced apoptotic cell death (Wu et al., 2011), a mitochondria-dependent effect of hydrogen peroxide. That hydrogen peroxide can be converted rapidly to other ROS (Veal et al., 2007) raises the possibility that multiple ROS may contribute to the symptoms in patients with various forms of chemotherapy-induced painful neuropathy. To confirm a role of Drp1 in ROS-induced mechanical hyperalgesia, we developed a model of hydrogen peroxide-induced hyperalgesia. We found that intradermal injection of hydrogen peroxide at the site of nociceptive testing produced a concentration-dependent decrease in mechanical nociceptive threshold. Also, the mechanical hyperalgesia induced by even the highest dose of hydrogen peroxide was markedly attenuated by mdivi-1.

Since we are now just beginning to understand how extracellular stimuli such as cytokines and growth factors modulate intracellular signaling to control oxidative stress and mitochondrial dynamics (Wu et al., 2011), we performed a preliminary evaluation of the role of Drp1 in mechanical hyperalgesia induced by pronociceptive cytokines and growth factors. We found that while mdivi-1 markedly attenuated mechanical hyperalgesia induced by the NO donor NOR-3, the growth factor GDNF, and $\mathrm{TNF} \alpha$, it did not significantly attenuate similar magnitude hyperalgesia induced by NGF or epinephrine. While the signaling pathways by which $\mathrm{NO}, \mathrm{TNF} \alpha$, and, potentially, other pronociceptive agonists activate Drp1 remains to be established, these findings suggest Drp1 to be involved in the generation of inflammatory as well as neuropathic pain.

Among its known functions in the cell, mitochondrial fission is upstream of caspase activation in the apoptotic signaling pathway (Li et al., 2010b). Drp1 activation of proapoptotic caspases, however, may not immediately produce cell death, as it can be reversed, in part, dependent on the magnitude of the insult (Barsoum et al., 2006). Thus, enhanced mitochondrial fission might be able to perturb neuronal function via apoptotic signaling pathways without leading to cell death. Of note in this regard, Cheng and Zochodne (2003) have shown that while there is a chronic elevation of caspase 3 , a proapoptotic caspase, in peripheral nerves of rats with diabetic painful peripheral neuropathy, there is no evidence of death of sensory neurons, even 1 year after induction of neuropathy. Furthermore, we have shown that caspase inhibitors can attenuate mechanical hyperalgesia in models of cisplatin and oxaliplatin painful peripheral neuropathy (Joseph and Levine, 2009). What determines whether activation of caspase signaling pathways induces apoptotic cell death versus enhancing neuronal activity remains to be determined.

Mitochondrial fission is a cytoskeleton-dependent phenomenon (Liu et al., 2009; Saks et al., 2010), and cytoskeleton is the major cellular target of cancer chemotherapeutic agents such as the vinca alkaloids and taxanes (Jordan and Wilson, 2004; Risinger et al., 2009). These cytoskeleton-targeted chemotherapeutic agents, which also cause mitochondrial fission (Wang et al., 2009) and an increase in ROS (Huang et al., 2005; Gogvadze et al., 2009), produce a small-fiber sensory painful peripheral neuropathy as a dose-limiting side effect (Postma et al., 1995; Polomano and Bennett, 2001; Quasthoff and Hartung, 2002). Of note, chemotherapeutic agents that target cytoskeleton affect mitochondrial movement, leading to excessive fission (Rovini et al., 2011). Whether Drp1 also contributes to pain in models of these forms of chemotherapy-induced painful neuropathy remains to be determined.

In conclusion, while accumulating evidence has implicated mitochondrial dysfunction, and especially oxidative stress, in the pathophysiology of several forms of painful peripheral neuropathy, especially those produced as side effects of chemotherapeutic drugs, antioxidants have been only marginally effective in the treatment of patients with diverse forms of painful peripheral neuropathy. Here we provide evidence that mitochondrial fission, an important component of mitochondrial dynamics that regulates multiple mitochondrial functions, may provide a more effective target for the treatment of neuropathic pain. While further studies are needed to more precisely define the role of mitochondrial fission in the pathophysiology of neuropathic pain, the recent development of small-molecule inhibitors of mitochondrial fission, such as mdivi-1, suggests that one may soon be able to therapeutically target mitochondrial fission in symptomatic peripheral neuropathies.

\section{References}

Aley KO, McCarter G, Levine JD (1998) Nitric oxide signaling in pain and nociceptor sensitization in the rat. J Neurosci 18:7008-7014.

Argyriou AA, Chroni E, Koutras A, Iconomou G, Papapetropoulos S, Polychronopoulos P, Kalofonos HP (2006) Preventing paclitaxel-induced peripheral neuropathy: a phase II trial of vitamin E supplementation. J Pain Symptom Manage 32:237-244.

Bailey SM (2003) A review of the role of reactive oxygen and nitrogen species in alcohol-induced mitochondrial dysfunction. Free Radic Res 37:585-596.

Barsoum MJ, Yuan H, Gerencser AA, Liot G, Kushnareva Y, Gräber S, Kovacs I, Lee WD, Waggoner J, Cui J, White AD, Bossy B, Martinou JC, Youle RJ, Lipton SA, Ellisman MH, Perkins GA, Bossy-Wetzel E (2006) Nitric oxide-induced mitochondrial fission is regulated by dynamin-related GTPases in neurons. EMBO J 25:3900-3911.

Berger AR, Arezzo JC, Schaumburg HH, Skowron G, Merigan T, Bozzette S, Richman D, Soo W (1993) 2', $3^{\prime}$-dideoxycytidine (ddC) toxic neuropathy: a study of 52 patients. Neurology 43:358-362.

Besse JL, Gadeyne S, Galand-Desmé S, Lerat JL, Moyen B (2009) Effect of vitamin $C$ on prevention of complex regional pain syndrome type I in foot and ankle surgery. Foot Ankle Surg 15:179-182.

Bogen O, Joseph EK, Chen X, Levine JD (2008) GDNF hyperalgesia is mediated by PLCgamma, MAPK/ERK, PI3K, CDK5 and Src family kinase signaling and dependent on the IB4-binding protein versican. Eur J Neurosci 28:12-19.

Brand MD (2010) The sites and topology of mitochondrial superoxide production. Exp Gerontol 45:466-472.

Brooks C, Wei Q, Cho SG, Dong Z (2009) Regulation of mitochondrial dynamics in acute kidney injury in cell culture and rodent models. J Clin Invest 119:1275-1285.

Bureković A, Terzić M, Alajbegović S, Vukojević Z, Hadzić N (2008) The role of alpha-lipoic acid in diabetic polyneuropathy treatment. Bosn J Basic Med Sci 8:341-345.

Cassidy-Stone A, Chipuk JE, Ingerman E, Song C, Yoo C, Kuwana T, Kurth MJ, Shaw JT, Hinshaw JE, Green DR, Nunnari J (2008) Chemical inhibition of the mitochondrial division dynamin reveals its role in Bax/Bakdependent mitochondrial outer membrane permeabilization. Dev Cell 14:193-204

Chen H, Chan DC (2009) Mitochondrial dynamics-fusion, fission, movement, and mitophagy-in neurodegenerative diseases. Hum Mol Genet 18:R169-R176.

Chen JY, Chang CY, Feng PH, Chu CC, So EC, Hu ML (2009) Plasma vita$\min \mathrm{C}$ is lower in postherpetic neuralgia patients and administration of vitamin $\mathrm{C}$ reduces spontaneous pain but not brush-evoked pain. Clin J Pain 25:562-569.

Cheng C, Zochodne DW (2003) Sensory neurons with activated caspase-3 survive long-term experimental diabetes. Diabetes 52:2363-2371.

Dalakas MC (2001) Peripheral neuropathy and antiretroviral drugs. J Peripher Nerv Syst 6:14-20.

Ferrari LF, Levine JD (2010) Alcohol consumption enhances antiretroviral 
painful peripheral neuropathy by mitochondrial mechanisms. Eur J Neurosci 32:811-818.

Ferrari LF, Bogen O, Levine JD (2010) Nociceptor subpopulations involved in hyperalgesic priming. Neuroscience 165:896-901.

Flatters SJ, Bennett GJ (2006) Studies of peripheral sensory nerves in paclitaxel-induced painful peripheral neuropathy: evidence for mitochondrial dysfunction. Pain 122:245-257.

Foster TS (2007) Efficacy and safety of alpha-lipoic acid supplementation in the treatment of symptomatic diabetic neuropathy. Diabetes Educ 33:111-117.

Gamelin E, Gamelin L, Bossi L, Quasthoff S (2002) Clinical aspects and molecular basis of oxaliplatin neurotoxicity: current management and development of preventive measures. Semin Oncol 29 [Suppl 15]:21-33.

Gane EJ, Weilert F, Orr DW, Keogh GF, Gibson M, Lockhart MM, Frampton CM, Taylor KM, Smith RA, Murphy MP (2010) The mitochondriatargeted anti-oxidant mitoquinone decreases liver damage in a phase II study of hepatitis C patients. Liver Int 30:1019-1026.

Gogvadze V, Orrenius S, Zhivotovsky B (2009) Mitochondria as targets for chemotherapy. Apoptosis 14:624-640.

Green K, Brand MD, Murphy MP (2004) Prevention of mitochondrial oxidative damage as a therapeutic strategy in diabetes. Diabetes 53 [Suppl 1]:S110-S118.

Huang R, Wallqvist A, Covell DG (2005) Anticancer metal compounds in NCI's tumor-screening database: putative mode of action. Biochem Pharmacol 69:1009-1039.

Inoue M, Sato EF, Nishikawa M, Park AM, Kira Y, Imada I, Utsumi K (2003) Mitochondrial generation of reactive oxygen species and its role in aerobic life. Curr Med Chem 10:2495-2505.

Jordan MA, Wilson L (2004) Microtubules as a target for anticancer drugs. Nat Rev Cancer 4:253-265.

Joseph EK, Levine JD (2006) Mitochondrial electron transport in models of neuropathic and inflammatory pain. Pain 121:105-114.

Joseph EK, Levine JD (2009) Comparison of oxaliplatin- and cisplatininduced painful peripheral neuropathy in the rat. J Pain 10:534-541.

Joseph EK, Levine JD (2010) Multiple PKCepsilon-dependent mechanisms mediating mechanical hyperalgesia. Pain 150:17-21.

Joseph EK, Chen X, Khasar SG, Levine JD (2004) Novel mechanism of enhanced nociception in a model of AIDS therapy-induced painful peripheral neuropathy in the rat. Pain 107:147-158.

Joseph EK, Chen X, Bogen O, Levine JD (2008) Oxaliplatin acts on IB4positive nociceptors to induce an oxidative stress-dependent acute painful peripheral neuropathy. J Pain 9:463-472.

Kallianpur AR, Hulgan T (2009) Pharmacogenetics of nucleoside reversetranscriptase inhibitor-associated peripheral neuropathy. Pharmacogenomics 10:623-637.

Kelland L (2007) The resurgence of platinum-based cancer chemotherapy. Nat Rev Cancer 7:573-584.

Kim S, Lee TJ, Park JW, Kwon TK (2008) Overexpression of cFLIPs inhibits oxaliplatin-mediated apoptosis through enhanced XIAP stability and Akt activation in human renal cancer cells. J Cell Biochem 105:971-979.

Lackner LL, Nunnari J (2010) Small molecule inhibitors of mitochondrial division: tools that translate basic biological research into medicine. Chem Biol 17:578-583.

Leene W, Roholl PJ, de Groot C (1976) Lymphocyte differentiation in the rabbit thymus. Ann Immunol (Paris) 127:911-921.

Lehky TJ, Leonard GD, Wilson RH, Grem JL, Floeter MK (2004) Oxaliplatin-induced neurotoxicity: acute hyperexcitability and chronic neuropathy. Muscle Nerve 29:387-392.

Li J, Zhou J, Li Y, Qin D, Li P (2010a) Mitochondrial fission controls DNA fragmentation by regulating endonuclease G. Free Radic Biol Med 49:622-631.

Li J, Li Y, Qin D, von Harsdorf R, Li P (2010b) Mitochondrial fission leads to Smac/DIABLO release quenched by ARC. Apoptosis 15:1187-1196.

Li J, Chen X, Xiao W, Ma W, Li T, Huang J, Liu X, Liang X, Tang S, Luo Y (2011) Mitochondria-targeted antioxidant peptide SS31 attenuates high glucose-induced injury on human retinal endothelial cells. Biochem Biophys Res Commun 404:349-356.

Lin MT, Beal MF (2006) Mitochondrial dysfunction and oxidative stress in neurodegenerative diseases. Nature 443:787-795.

Liu X, Weaver D, Shirihai O, Hajnóczky G (2009) Mitochondrial "kiss-andrun": interplay between mitochondrial motility and fusion-fission dynamics. EMBO J 28:3074-3089.
Malik-Hall M, Dina OA, Levine JD (2005) Primary afferent nociceptor mechanisms mediating NGF-induced mechanical hyperalgesia. Eur J Neurosci 21:3387-3394.

Mijnhout GS, Alkhalaf A, Kleefstra N, Bilo HJ (2010) Alpha lipoic acid: a new treatment for neuropathic pain in patients with diabetes? Neth J Med 68:158-162.

Mondal D, Pradhan L, Ali M, Agrawal KC (2004) HAART drugs induce oxidative stress in human endothelial cells and increase endothelial recruitment of mononuclear cells: exacerbation by inflammatory cytokines and amelioration by antioxidants. Cardiovasc Toxicol 4:287-302.

Murphy MP, Smith RA (2007) Targeting antioxidants to mitochondria by conjugation to lipophilic cations. Annu Rev Pharmacol Toxicol 47:629-656.

Osio M, Muscia F, Zampini L, Nascimbene C, Mailland E, Cargnel A, Mariani C (2006) Acetyl-1-carnitine in the treatment of painful antiretroviral toxic neuropathy in human immunodeficiency virus patients: an open label study. J Peripher Nerv Syst 11:72-76.

Otera H, Mihara K (2011) Molecular mechanisms and physiologic functions of mitochondrial dynamics. J Biochem 149:241-251.

Pace A, Giannarelli D, Galiè E, Savarese A, Carpano S, Della Giulia M, Pozzi A, Silvani A, Gaviani P, Scaioli V, Jandolo B, Bove L, Cognetti F (2010) Vitamin E neuroprotection for cisplatin neuropathy: a randomized, placebo-controlled trial. Neurology 74:762-766.

Parada CA, Yeh JJ, Joseph EK, Levine JD (2003) Tumor necrosis factor receptor type-1 in sensory neurons contributes to induction of chronic enhancement of inflammatory hyperalgesia in rat. Eur J Neurosci 17:1847-1852.

Peltier AC, Russell JW (2002) Recent advances in drug-induced neuropathies. Curr Opin Neurol 15:633-638.

Polomano RC, Bennett GJ (2001) Chemotherapy-evoked painful peripheral neuropathy. Pain Med 2:8-14.

Postma TJ, Vermorken JB, Liefting AJ, Pinedo HM, Heimans JJ (1995) Paclitaxel-induced neuropathy. Ann Oncol 6:489-494.

Quasthoff S, Hartung HP (2002) Chemotherapy-induced peripheral neuropathy. J Neurol 249:9-17.

Risinger AL, Giles FJ, Mooberry SL (2009) Microtubule dynamics as a target in oncology. Cancer Treat Rev 35:255-261.

Rocha M, Hernandez-Mijares A, Garcia-Malpartida K, Bañuls C, Bellod L, Victor VM (2010) Mitochondria-targeted antioxidant peptides. Curr Pharm Des 16:3124-3131.

Rovini A, Savry A, Braguer D, Carré M (2011) Microtubule-targeted agents: when mitochondria become essential to chemotherapy. Biochim Biophys Acta 1807:679-688.

Saks V, Guzun R, Timohhina N, Tepp K, Varikmaa M, Monge C, Beraud N, Kaambre T, Kuznetsov A, Kadaja L, Eimre M, Seppet E (2010) Structure-function relationships in feedback regulation of energy fluxes in vivo in health and disease: mitochondrial interactosome. Biochim Biophys Acta 1797:678-697.

Sima A (2007) Acetyl-L-carnitine in diabetic polyneuropathy: experimental and clinical data. CNS Drugs 21 [Suppl 1]:13-23.

Skjelbakken T, Valen G, Vaage J (1996) Perfusing isolated rat hearts with hydrogen peroxide: an experimental model of cardiac dysfunction caused by reactive oxygen species. Scand J Clin Lab Invest 56:431-439.

Skuta G, Fischer GM, Janaky T, Kele Z, Szabo P, Tozser J, Sumegi B (1999) Molecular mechanism of the short-term cardiotoxicity caused by $2^{\prime}, 3^{\prime}$ dideoxycytidine (ddC): modulation of reactive oxygen species levels and ADP-ribosylation reactions. Biochem Pharmacol 58:1915-1925.

Smith RA, Murphy MP (2011) Mitochondria-targeted antioxidants as therapies. Discov Med 11:106-114.

Stone JR, Yang S (2006) Hydrogen peroxide: a signaling messenger. Antioxid Redox Signal 8:243-270.

Su B, Wang X, Zheng L, Perry G, Smith MA, Zhu X (2010) Abnormal mitochondrial dynamics and neurodegenerative diseases. Biochim Biophys Acta 1802:135-142.

Summer GJ, Romero-Sandoval EA, Bogen O, Dina OA, Khasar SG, Levine JD (2008) Proinflammatory cytokines mediating burn-injury pain. Pain 135:98-107.

Tan E, Janssen A, Roestenberg P, van den Heuvel L, Goris R, Rodenburg R (2011) Mitochondrial dysfunction in muscle tissue of complex regional pain syndrome type I patients. Eur J Pain. Advance online publication. Retrieved July 14, 2011. PMID: 21262583. 
Tanaka A, Youle RJ (2008) A chemical inhibitor of DRP1 uncouples mitochondrial fission and apoptosis. Mol Cell 29:409-410.

Tournigand C, André T, Achille E, Lledo G, Flesh M, Mery-Mignard D, Quinaux E, Couteau C, Buyse M, Ganem G, Landi B, Colin P, Louvet C, de Gramont A (2004) FOLFIRI followed by FOLFOX6 or the reverse sequence in advanced colorectal cancer: a randomized GERCOR study. J Clin Oncol 22:229-237.

Vanotti A, Osio M, Mailland E, Nascimbene C, Capiluppi E, Mariani C (2007) Overview on pathophysiology and newer approaches to treatment of peripheral neuropathies. CNS Drugs 21 [Suppl 1]:3-12.

Veal EA, Day AM, Morgan BA (2007) Hydrogen peroxide sensing and signaling. Mol Cell 26:1-14.

Wang JX, Li Q, Li PF (2009) Apoptosis repressor with caspase recruitment domain contributes to chemotherapy resistance by abolishing mitochondrial fission mediated by dynamin-related protein-1. Cancer Res 69:492-500.
Westermann B (2010) Mitochondrial fusion and fission in cell life and death. Nat Rev Mol Cell Biol 11:872-884.

Wu S, Zhou F, Zhang Z, Xing D (2011) Mitochondrial oxidative stress causes mitochondrial fragmentation via differential modulation of mitochondrial fission-fusion proteins. FEBS J 278:941-954.

Yu T, Sheu SS, Robotham JL, Yoon Y (2008) Mitochondrial fission mediates high glucose-induced cell death through elevated production of reactive oxygen species. Cardiovasc Res 79:341-351.

Ziegler D, Nowak H, Kempler P, Vargha P, Low PA (2004) Treatment of symptomatic diabetic polyneuropathy with the antioxidant alpha-lipoic acid: a meta-analysis. Diabet Med 21:114-121.

Ziegler D, Ametov A, Barinov A, Dyck PJ, Gurieva I, Low PA, Munzel U, Yakhno N, Raz I, Novosadova M, Maus J, Samigullin R (2006) Oral treatment with alpha-lipoic acid improves symptomatic diabetic polyneuropathy: the SYDNEY 2 trial. Diabetes Care 29:2365-2370. 